\title{
Case report of relaxation experience to support body-mediated communication in mother-child interactions compared by free play
}

Yuka Mizuno*, Akari Fujii, Xinyi Liu, Russell Sarwar Kabir, Yutaka Haramaki

Graduate School of Education, Hiroshima University, Higashihiroshima, Hiroshima, Japan

\section{Background:}

Mother-child relationships are made up of cumulative interactions.

Developmental process

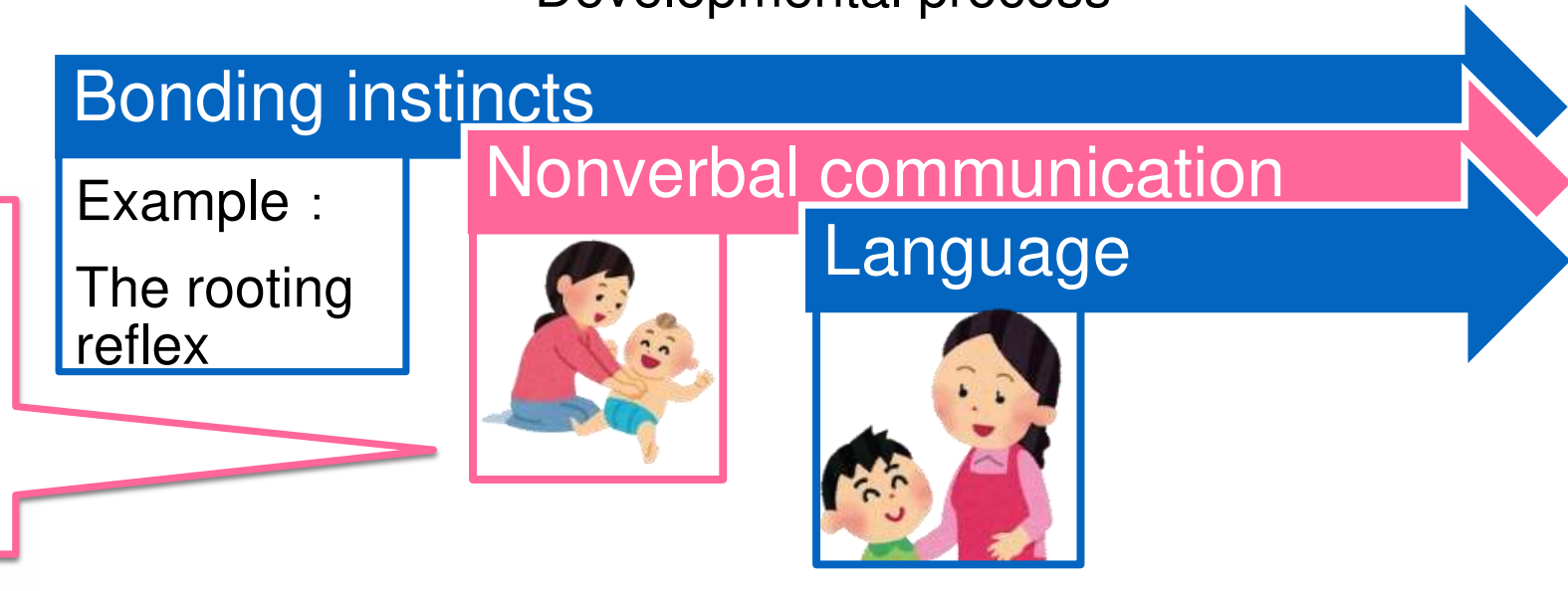

Using a recently described psychotherapeutic approach known as Self-Active Relaxation Therapy (SART ; Ohono, 2005) that draws attention to body-mediated communication through a set system of relaxation tasks, we conducted a case study designed to gain insight into the dynamics of reciprocal understanding between mothers and their children.

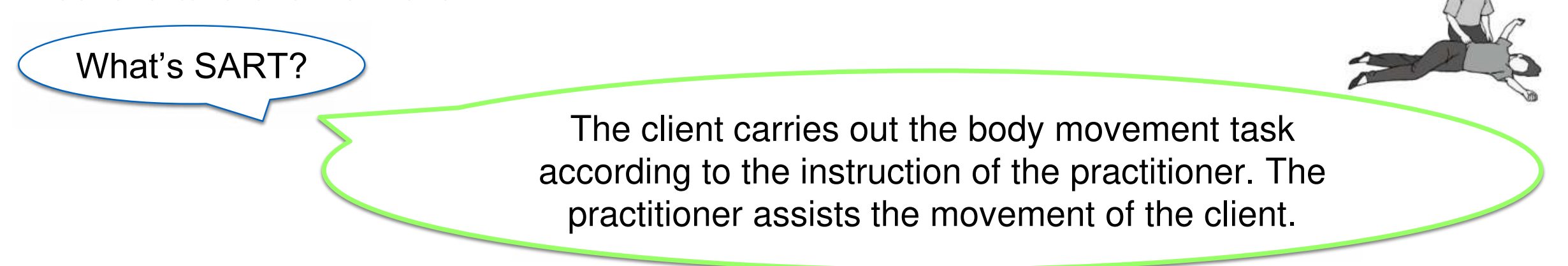

\section{Methods:}

The focus of behavioral description was set to the interactions between one mother (28 years old) and her son ( 2 years and 5 months old).

The study was conducted in an ABA research design at the home of mother and child as a naturalistic setting .

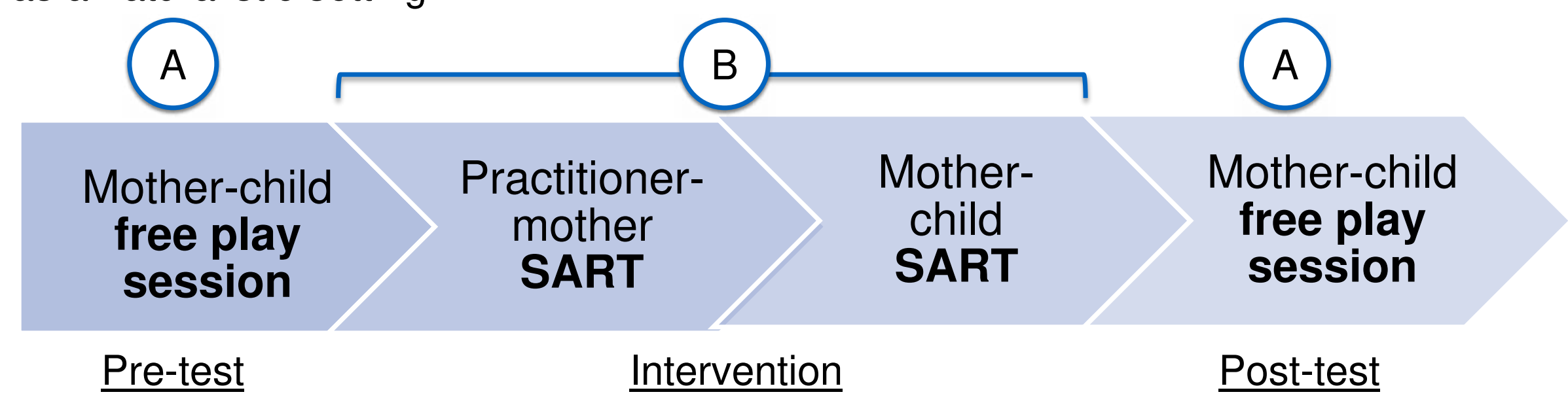

Three researchers recorded video of the whole session (1 hour) and described changes of the mother-child interactions from pre-test to post-test.

The practitioner who is one of the researchers in the B-phase had two years of SART training.

\section{Findings:}

Qualitative analysis and comparison of the free play sessions revealed some observable changes in behavioral tendencies.

The pre-SART A phase session indicated that the child generally led the play session.

In the first B phase, it was observed that the mother gradually moved her body actively and at her own pace.

In the second B phase, the mother communicated the movements and demonstrated them with incremental understanding of the self-activeness of her child, and the child participated in the movements actively in response.

In the post-SART A phase session, the mother was notably invited by the son to play together while illustrating some mutually raised and recognized awareness about each other during play.

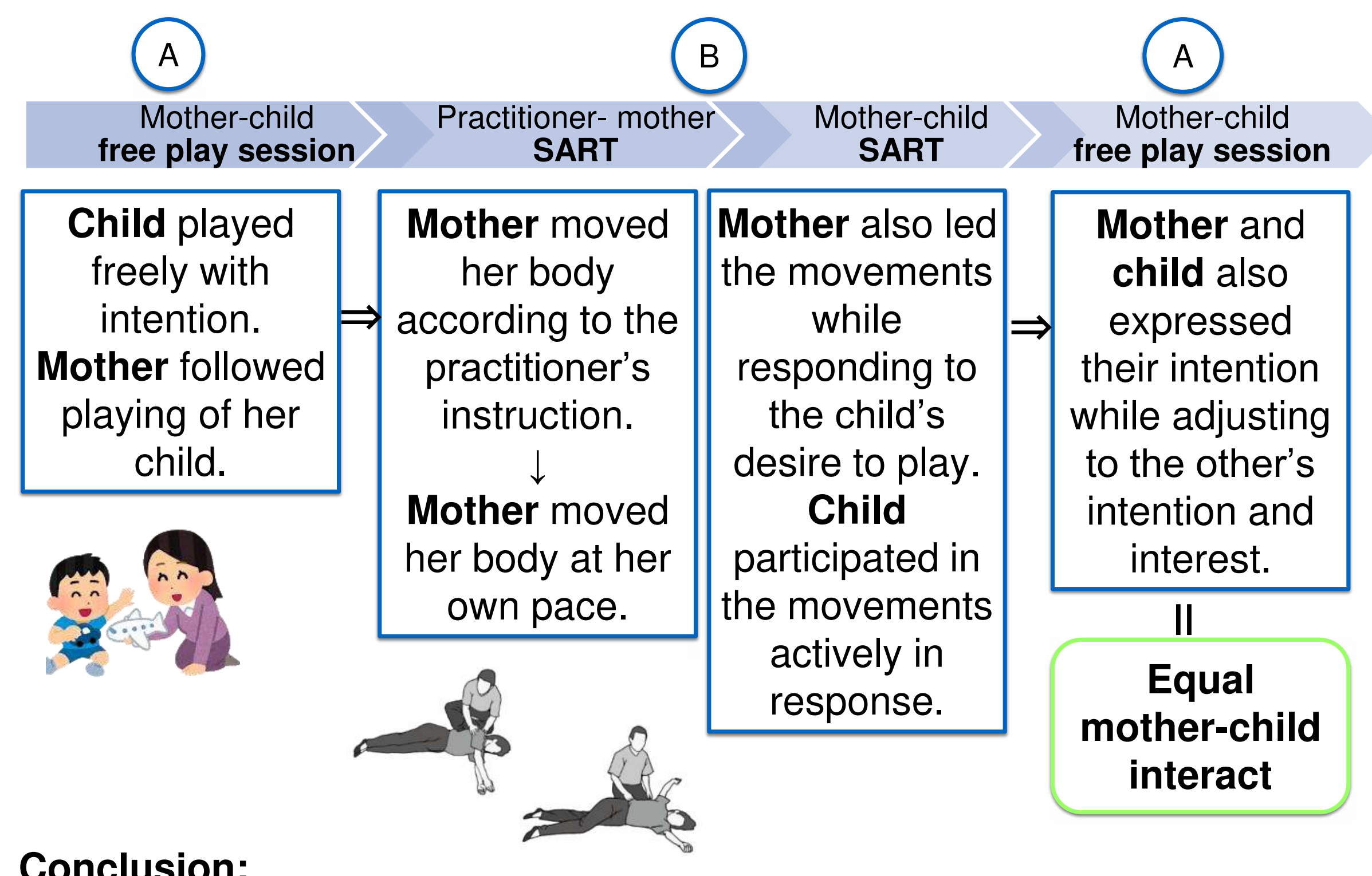

From the behavioral changes in free play tendencies, this case report provides some initial observation in a support setting that experience with SART as a body-mediated communication tool facilitated some positive mutual interactions between a mother and child. 